\title{
Effects of lumican expression on the apoptosis of scleral fibroblasts: In vivo and in vitro experiments
}

\author{
JINSONG WU ${ }^{1}$, YANZHI ZHAO ${ }^{2}$, YANMEI FU ${ }^{1}$, SHURONG LI $^{1}$ and XU ZHANG ${ }^{3}$ \\ ${ }^{1}$ Department of Pediatric Ophthalmology, The Second Affiliated Hospital of Nanchang University, Nanchang, Jiangxi 330006; \\ ${ }^{2}$ The First Clinical Medical College; ${ }^{3}$ The Affiliated Eye Hospital, Nanchang University, Nanchang, Jiangxi 330000, P.R. China
}

Received February 12, 2020; Accepted September 15, 2020

DOI: $10.3892 /$ etm.2021.9926

\begin{abstract}
Lumican serves an important role in the maintenance of sclera biomechanical properties. However, whether lumican expression is altered in myopia and the mechanisms of action involved are unknown. In the present study, the expression of lumican in cultured scleral fibroblasts and in the scleral tissue of a rat model of form-deprivation myopia was assessed. It was confirmed that diopter was decreased, whereas axial length was increased in modeled eyes relative to normal control eyes, indicating that the model of myopia was successfully established. These pathologic changes were accompanied by the upregulation of lumican and tissue inhibitor of metalloproteinases (TIMP)-2, as well as the downregulation of matrix metalloproteinase (MMP)-2 and MMP-14. The same trends in TIMP-2, MMP-2 and MMP-14 expression were observed when lumican was overexpressed in cultured scleral fibroblasts. Additionally, cell proliferation decreased whereas apoptosis increased compared with those of control cells. Inhibiting lumican expression had no effect on cell proliferation or apoptosis, but stimulated the expression of MMP-2 and MMP-14 while decreasing that of TIMP-2. The results suggested that lumican overexpression contributed to myopia by promoting apoptosis in scleral fibroblasts via the modulation of TIMP-2, MMP-2 and MMP-14 expression.
\end{abstract}

\section{Introduction}

Myopia is one of the most common eye diseases, affecting $27 \%$ of the population worldwide in 2010 (1). The sclera, which contributes to the regulation of refractive status, is a critical structure involved in the development of myopia (2). Clinical and experimental studies have demonstrated that changes in the biochemical and mechanical properties of the

Correspondence to: Dr Jinsong Wu, Department of Pediatric Ophthalmology, The Second Affiliated Hospital of Nanchang University, 1 Minde Road, Nanchang, Jiangxi 330006, P.R. China E-mail: wujinsong628@163.com

Key words: myopia, lumican, scleral fibroblasts, matrix metalloproteinase sclera during the development of myopia can induce scleral remodeling and lengthening of the ocular axis (3); with scleral remodeling serving an important role in emmetropia and eye development (4). Fibroblasts are the major cell type within the sclera and the main cells affected in myopia (5). Structural and functional abnormalities in scleral fibroblasts have been implicated in various pathologies (6).

The lumican gene encodes leucine glycan, a regulator of scleral development that is present at high levels in the extracellular matrix. The protein core interacts with collagen molecules to regulate the diameter of collagen fibers (7) and the inter-fiber space (8). Lumican has been shown to inhibit the lateral aggregation of collagen molecules and limit the diameter of collagen fibers so as to maintain the biomechanical properties of the sclera, such as elasticity and tension $(9,10)$. Mutations to the lumican gene are thought to be a cause of myopia, as demonstrated by loss-of-function experiments in animal models (11). However, whether lumican expression is altered in myopia and the mechanisms of action involved are unclear. The present study aimed to address these areas using cultured scleral fibroblasts and a rat model of myopia.

\section{Materials and methods}

Animals. Male Sprague-Dawley rats (21 days old; $\mathrm{n}=20$; $120 \pm 5 \mathrm{~g}$ ) were purchased from Shanghai Laboratory Animal Research Center [license no. scxk (Lu) 2018-0006] and housed under specific pathogen-free conditions at $23 \pm 2^{\circ} \mathrm{C}$, at a relative humidity of $45-65 \%$ and under a $12 \mathrm{~h}$ light/dark cycle. The animals were fed rodent pellets and water ad libitum. For the cell culture, an additional 6 male newborn rats $(5 \mathrm{~g})$ were also purchased from Shanghai Laboratory Animal Research Center [license no. scxk (Lu) 2018-0006] All experimental protocol was approved by the Ethics Committee of the Second Affiliated Hospital of Nanchang University.

Establishment of the myopia model. The myopia model was established as previously described (12). Rats were anesthetized with isoflurane (5\% for induction and $2 \%$ for maintenance) and immobilized on a surgical table in the prone position. The hair around the right eye was shaved with a razor and the skin sterilized with $1 \%$ iodophor. A $2-\mathrm{mm}$ long incision was made with ophthalmic scissors, $3 \mathrm{~mm}$ inside the right eye, outside 
the outer canthus and sutured with a 6-0 needle. The tip of the needle was inserted through the incision of the outer canthus, lower eyelid and inner canthus of the eye. The start and end points of the suture were tightened until the eyelid was closed. The uninjured left eye served as the control. Experiments using the rats were performed 8 weeks after surgery.

Diopter detection. Tropicamide $(0.5 \%, 5 \mu 1$, each eye) was applied to the eyes as drops. After $3 \mathrm{~min}$, the pupils were sufficiently dilated, with a suitable working distance. The 3 hole/1 line was maintained with a strip light detector and diopter was determined while moving the light band back and forth. The final diopter was calculated by adding and subtracting the positive and negative diopters of different degrees for neutralization according to the following formula: Final diopter $=($ degrees for examined eye $)-[$ working distance mirror (d)].

Axial measurement. Rats were anesthetized with isoflurane $(5 \%)$ and then sacrificed by decapitation. The eyeball was dissected, placed on a sterile gauze moistened with normal saline on ice and the axis was measured with Vernier calipers. The average value of 3 measurements was reported.

Hematoxylin and eosin $(H \& E)$ staining. Sclera tissue samples were fixed in $4 \%$ paraformaldehyde for $30 \mathrm{~min}$ at room temperature, then dehydrated in 70,80 and 90\% ethanol solutions. The samples were then immersed in pure ethanol and xylene for $15 \mathrm{~min}$, xylene I for $15 \mathrm{~min}$ and xylene II for $15 \mathrm{~min}$ at room temperature until the tissue became transparent. This was followed by immersion in a mixture of xylene and paraffin for $15 \mathrm{~min}$ and paraffin I and paraffin II for 50-60 min. Finally, the samples were embedded in paraffin at room temperature for $5 \mathrm{~min}$ and sectioned at a thickness of $10 \mu \mathrm{m}$. The sections were collected on a slide and baked at $60^{\circ} \mathrm{C}$ for $2 \mathrm{~h}$, deparaffinized and rehydrated, then stained with hematoxylin for $3 \mathrm{~min}$ and eosin for $3 \mathrm{~min}$ at room temperature. The slides were sealed and at least four fields of each slide were imaged using a light microscope (magnification, x200; BX51; Olympus Corporation).

Immunohistochemistry. Eyeball tissue sections were prepared as aforementioned followed by blocking in $5 \%$ bovin serum albumin (Hyclone; GE Healthcare Life Science) at room temperature for $2 \mathrm{~h}$ and incubated overnight at $4^{\circ} \mathrm{C}$ with monoclonal antibodies against MMP2 (1:250; cat. no. AF0577), tissue inhibitor of metalloproteinases (TIMP)-2 (1:200; cat. no. AF7008; each from Affinity Biosciences), MMP-14 (1:100; cat. no. ab51074) and lumican (1:100, cat. no. ab168348; each purchased from Abcam), followed by horseradish peroxidase (HRP)-conjugated goat anti-rabbit IgG (1:10,000; cat. no. A16104; Thermo Fisher Scientific, Inc.) or Alexa Fluor 593 goat anti-mouse IgG (1:200; cat. no. A-11001; Thermo Fisher Scientific, Inc.) for $30 \mathrm{~min}$ at room temperature. Immunoreactivity was visualized by incubation with 3,3'-diaminobenzidine for $3 \mathrm{~min}$ at room temperature. At least four fields were taken from each image using a light microscope (magnification, x200; BX51, Olympus Corporation). The grey value of the staining was analyzed by Image-Pro Plus software v6.0 (National Institutes of Health).
Transmission electron microscopy. Scleral tissue samples were fixed with $2.5 \%$ glutaraldehyde at room temperature for $30 \mathrm{~min}$ and then dehydrated, embedded in epoxy resin at $60^{\circ} \mathrm{C}$ for $24 \mathrm{~h}$ and sectioned at a thickness of $100 \mathrm{~nm}$. The sections were stained with $3 \%$ uranyl acetate and lead citrate at room temperature for $5 \mathrm{~min}$, and then imaged by transmission electron microscopy (magnification, $\mathrm{x} 1,000 ; 80 \mathrm{kV}$; JEM-1230; JEOL, Ltd.).

Cell culture. The Scleral tissue was collected from six newborn rats and cut into $1 \times 1 \mathrm{~mm}$ sections that were digested in $0.1 \%$ type II collagenase at $37^{\circ} \mathrm{C}$ for $30 \mathrm{~min}$. After filtering through a $70-\mu \mathrm{m}$ mesh, the cells were collected by centrifugation $\left(1,000 \mathrm{x} \mathrm{g}\right.$ for $5 \mathrm{~min}$ at $\left.4^{\circ} \mathrm{C}\right)$, resuspended in RPMI-1640 medium containing $20 \% \mathrm{FBS}$ and cultured at $37^{\circ} \mathrm{C}$ and $5 \% \mathrm{CO}_{2}$, to make primary scleral fibroblast cultures.

Cell transfection. Lumican overexpression or silencing constructs $(2 \mu \mathrm{g} / \mu \mathrm{l})$ and a negative control were produced by Shanghai GenePharma Co., Ltd using pcDNA3.1 plasmid (Invitrogen; Thermo Fisher Scientific, Inc.). Scleral fibroblasts were cultured until they reached $70 \%$ confluence, then transfected at room temperature with $1.25 \mu \mathrm{g}$ of pcDNA3.1 plasmid (Invitrogen; Thermo Fisher Scientific, Inc.) using $5 \mu$ l Lipofectamine ${ }^{\circledR} 3000$ (Invitrogen; Thermo Fisher Scientific, Inc.). After $2 \mathrm{~h}$, the culture medium was refreshed and the cells were divided into the following five groups: Untransfected (negative control), lumican overexpression construct ( $2 \mu \mathrm{g} / \mu \mathrm{l}$, Shanghai GenePharma Co., Ltd), empty vector (negative control), small interfering (si)RNA construct, and scrambled siRNA construct (negative control). After $48 \mathrm{~h}$, the cells were used to evaluate cell proliferation, apoptosis and protein expression. The siRNA sequences for lumican are listed in Table I.

Reverse transcription-quantitative $(q) P C R$. RNA was extracted from cells using TRIzol ${ }^{\circledR}$ (Thermo Fisher Scientific, Inc.) and cDNA was synthesized at $30^{\circ} \mathrm{C}$ for $10 \mathrm{~min}$ according to the instructions of the reverse transcriptase kit (CoWin Biosciences). cDNA was used as the template for qPCR, which was performed on a 7500 Fast Real-Time PCR System (Applied Biosystems). The level of $\beta$-actin was used as the internal reference to calculate the mRNA expression levels of, MMP-2, MMP-14 and TIMP-2. The qPCR reaction consisted of $9.5 \mu 1$ RNase-free $\mathrm{dH}_{2} \mathrm{O}, 1 \mu \mathrm{l} \mathrm{cDNA} / \mathrm{DNA}, 2 \mu \mathrm{l}$ primers and $12.5 \mu \mathrm{l}$ 2X UltraSYBR mix (cat. no. A25778; Thermo Fisher Scientific, Inc.). The cycling protocol was as follows: Pre-denaturation at $95^{\circ} \mathrm{C}$ for $10 \mathrm{~min}, 40$ cycles of $95^{\circ} \mathrm{C}$ for $10 \mathrm{sec}, 54.3^{\circ} \mathrm{C}$ for $30 \mathrm{sec}$ and $72^{\circ} \mathrm{C}$ for $30 \mathrm{sec}$. The target gene expression was normalized to $\beta$-actin as previously described (13). Primer sequences are listed in Table II.

Western blotting. Total protein was extracted from scleral tissues or cultured scleral fibroblasts. After determining the concentration using the BCA method, the proteins $(20 \mu \mathrm{g})$ were separated by $12 \%$ SDS-PAGE and transferred to a PVDF membrane. Non-specific antibody binding was blocked by incubating the membrane in $5 \%$ non-fat milk at room temperature for $2 \mathrm{~h}$. The membrane was then incubated overnight at $4{ }^{\circ} \mathrm{C}$ with antibodies against MMP-2 (1:1,000), MMP-14 (1:1,000), TIMP-2 (1:1,000), 
Table I. siRNA sequences.

SiRNA siRNA sequence (5'-3')

Lumican-siRNA1

Lumican-siRNA2

Lumican-siRNA3

Scrambled siRNA (siRNA-NC)
ACAAUAAGCUCAAGAGUAUTTAUACUCUUGAGCUUAUUGUTT UGAAGAAGCUGCAUAUAAATTUUUAUAUGCAGCUUCUUCATT ACUCCAAGAUCAAAGGAAATTUUUCCUUUGAUCUUGGAGUTT UUCUCCGAACGUGUCACGUTTACGUGACACGUUCGGAGAATT

$\mathrm{NC}$, negative control; $\mathrm{Si}$, small interfering.

Table II. Primer sequences.

\begin{tabular}{llccc}
\hline Primer & \multicolumn{1}{c}{ Sequence $\left(5^{\prime}-3^{\prime}\right)$} & $\begin{array}{c}\text { Primer length } \\
(\mathrm{bp})\end{array}$ & $\begin{array}{c}\text { Product length } \\
(\mathrm{bp})\end{array}$ & $\begin{array}{c}\text { Annealing temperature } \\
\left({ }^{\circ} \mathrm{C}\right)\end{array}$ \\
\hline Lumican F & GCCTTTGAGAATGTAACGGAT & 21 & 169 & 57.1 \\
Lumican R & CTTGTAGGGACTTTGGGAGC & 20 & & 58.1 \\
MMP-2 F & AGGACACCCTCAAGAAGATGC & 21 & 134 & \\
MMP-2 R & GCGGGGAAAGAAGTTGTAGTT & 21 & \multirow{2}{*}{58.4} \\
MMP-14 F & GCAGTATGGCTACCTACCTCC & 21 & & \\
MMP-14 R & CTTGCCTGTCACTTGTAAACC & 21 & 212 & 56.6 \\
TIMP-2 F & GCAACCCCATCAAGAGGA & 18 & & 56.5 \\
TIMP-2 R & CCAGGGCACAATAAAGTCAC & 20 & 90 & \\
$\beta$-actin F & ACGGTCAGGTCATCACTATC & 20 & & \\
$\beta$-actin R & TGCCACAGGATTCCATACC & 19 &
\end{tabular}

bp, base pairs; F, forwards; R, reverse; TIMP, tissue inhibitor of metalloproteinases.

lumican(1:1,000) and GAPDH(1:3,000; cat.no.ab8245; Abcam), followed by incubation with HRP-conjugated anti-rabbit IgG (1:10,000; cat. no. A16104SAMPLE; Thermo Fisher Scientific, Inc.) at room temperature for $2 \mathrm{~h}$. An enhanced chemiluminescence reagent kit (cat. no. WP20005; Thermo Fisher Scientific, Inc.) was used to visualize immunoreactivity. The blots were scanned with a ChemiDoc XRS imaging system (Bio-Rad Laboratories, Inc.) and signal intensity was analyzed with Quantity One v1.4.6 software (Bio-Rad Laboratories, Inc.) as previously described (14).

Cell Counting Kit (CCK)- 8 assay. Transfected cells were digested, resuspended at a density of $5 \times 10^{3}$ cells/well and counted under a light microscope (magnification, x200; BX51; Olympus Corporation). After $24 \mathrm{~h}, 10 \mu \mathrm{l} \mathrm{CCK}-8$ reagent (cat. no. C0037, Beyotime Institute of Biotechnology) was added to each well, followed by incubation for $2 \mathrm{~h}$ at $37^{\circ} \mathrm{C}$ according to the instruction of the kit. The absorbance of each well was measured at a wavelength of $450 \mathrm{~nm}$ using a microplate reader.

Flow cytometry. A total of $3 \times 10^{6}$ cells were collected from each group and centrifuged with $1 \mathrm{ml}$ PBS at 1,500 $\mathrm{x} g$ for $3 \mathrm{~min}$ at room temperature. After two washes with 0.1 M PBS, cells (in $1 \mathrm{ml}$ ) were incubated in the dark for $10 \mathrm{~min}$ with $3 \mu \mathrm{l}$ FITC-annexin $\mathrm{V}$ and $5 \mu \mathrm{l}$ propidium iodide at room temperature (cat. no. C1062M, Beyotime Institute of Biotechnology).
Apoptotic cells were detected by NovoCyte ${ }^{\mathrm{TM}}$ flow cytometer (NovoCyte 2060R; ACEA Bioscience, Inc.) and analyzed using FlowJo v10 (FlowJo, LLC).

Statistical analysis. Data are expressed as the mean \pm SD and were analyzed using SPSS v19.0 software (IBM Corp.). The control and modeled eyes were from the same animals; therefore, paired Student's t-tests were used to analyze the differences. When there were $\geq 3$ groups, the differences were evaluated using one-way ANOVA followed by a post-hoc Tukey's test. $\mathrm{P}<0.05$ was considered to indicate a statistically significant difference.

\section{Results}

Evaluation of the form-deprivation myopia model. In myopia model eyes, the diopter was decreased (Fig. 1A) and axial length was increased $(\mathrm{P}<0.05$; Fig. 1B) compared with control eyes, indicating that the model was successfully established. Furthermore, histologic analysis via H\&E staining revealed that in the control eyes, the collagen fibers of scleral tissue were evenly distributed and had an orderly arrangement, with little extracellular matrix. In contrast, in myopia model eyes, the sclera was thinner and collagen fibers were more sparse and disorganized; fractured fibers were also observed (Fig. 1C). Electron microscopy analysis confirmed the presence of partly disintegrated and vacuolated collagen fibers with variable 

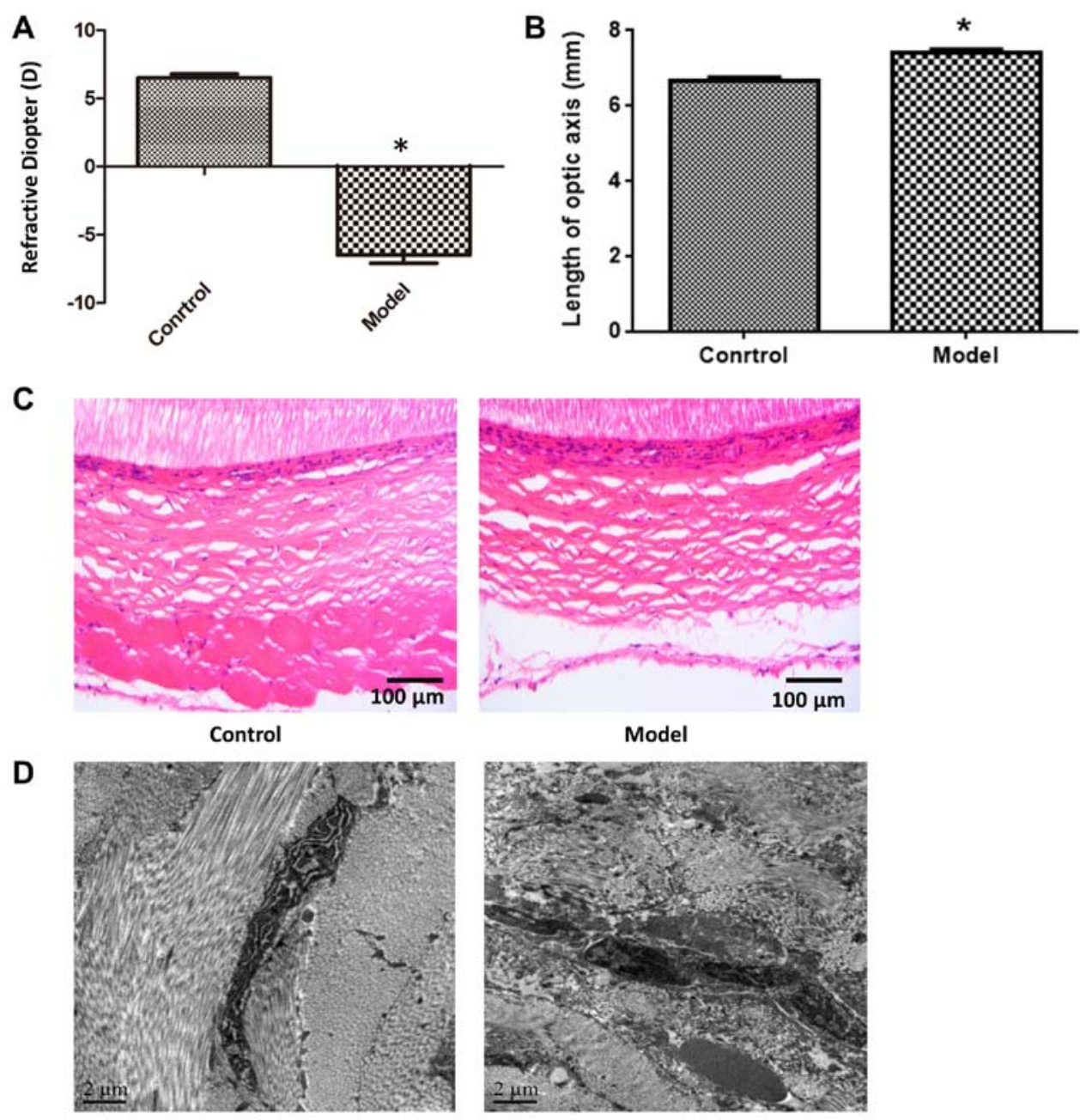

Control

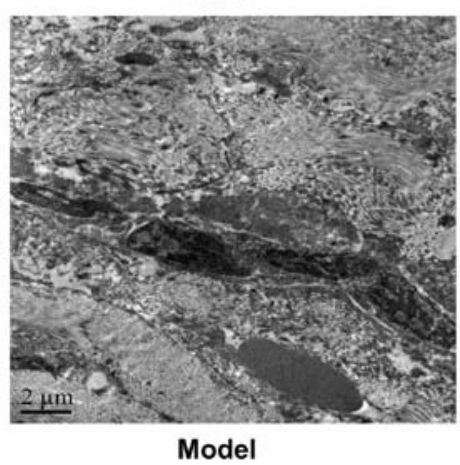

Figure 1. Abnormal diopter and axial length, and pathologic changes in scleral tissue in a model of form-deprivation myopia. (A) D value was reduced in model eyes and (B) the optic axis was increased in the model eyes. (C) Pathologic changes in scleral tissue were observed by hematoxylin and eosin staining. (D) Ultrastructural analysis of scleral tissue was performed via electron microscopy. ${ }^{*} \mathrm{P}<0.05$ vs. control eyes (paired t-tests). D, diopter.

thickness and disordered arrangement in the posterior sclera, as well as the enlargement of inter-fiber space (Fig. 1D).

Expression of lumican, MMP-2, MMP-14 and TIMP-2 in rat scleral tissue. The expression levels of lumican, MMP-2, MMP-14 and TIMP-2 in scleral tissue was evaluated at the mRNA and protein level. Compared with control eyes, lumican and TIMP-2 transcript levels were increased, whereas MMP-2 and MMP-14 levels were lower in the myopia model (Fig. 2A). Western blot analysis revealed that lumican expression was increased in myopia model eyes compared with control eyes (Fig. 2B). Similar trends were observed by immunohistochemistry, with higher expression of lumican and TIMP-2 and lower expression of MMP-2 and MMP-14 observed in myopia model eyes relative to control eyes (Fig. 2C and D).

Lumican overexpression and knockdown in cultured scleral fibroblasts. Vimentin expression was detected in primary cultured scleral fibroblasts (Fig. 3A). Compared with the control group, lumican expression at the mRNA (Fig. 3B) and protein (Fig. 3C) levels were higher in the lumican overexpression group, whereas lumican knockdown resulted in a loss of expression (Fig. 3D and E). The lumican-siRNA2 construct had the highest knockdown efficiency and was used for subsequent experiments.

Lumican overexpression reduces cell viability and promotes apoptosis in scleral fibroblasts. Compared with scleral fibroblasts transfected with the empty vector, viability was reduced in cells overexpressing lumican (Fig. 4A). However, siRNA-mediated lumican knockdown had no effect on cell viability. Similarly, apoptosis was enhanced relative to the control group by lumican overexpression but was unaffected by lumican knockdown (Fig. 4B and C).

Lumican overexpression or knockdown alters MMP-2, MMP-14 and TIMP-2 expression levels in rat scleral fibroblasts. Compared with the control group, MMP-2 and MMP-14 levels were reduced while TIMP-2 levels were increased in cells overexpressing lumican (Fig. 5). In contrast, MMP-2 and MMP-14 were upregulated whereas TIMP-2 was downregulated in cells transfected with lumican siRNA compared with those transfected with the scrambled control siRNA. 
A

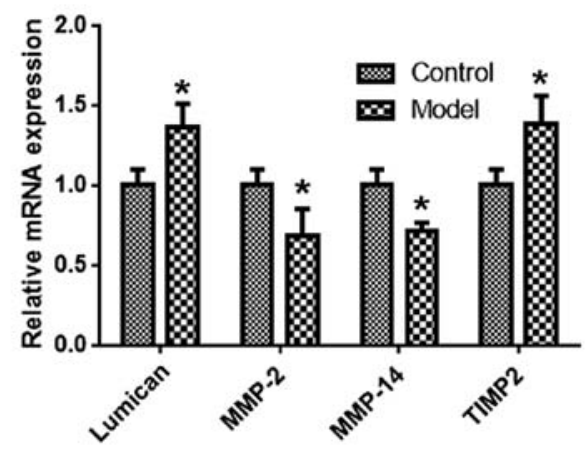

C
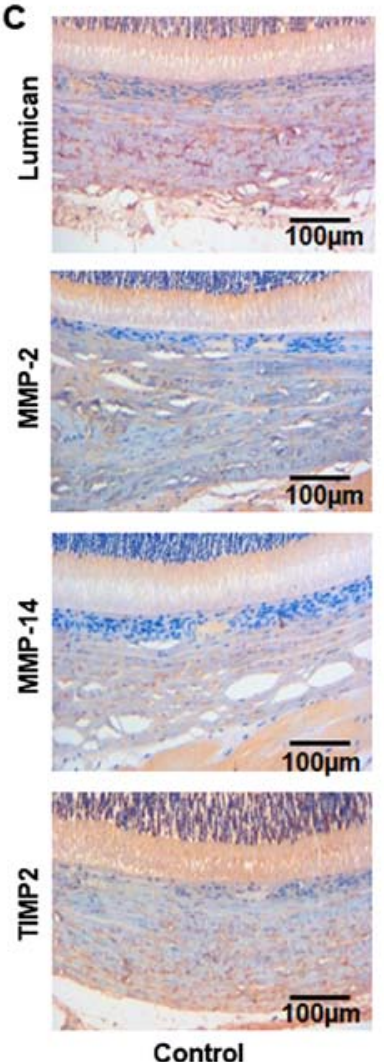
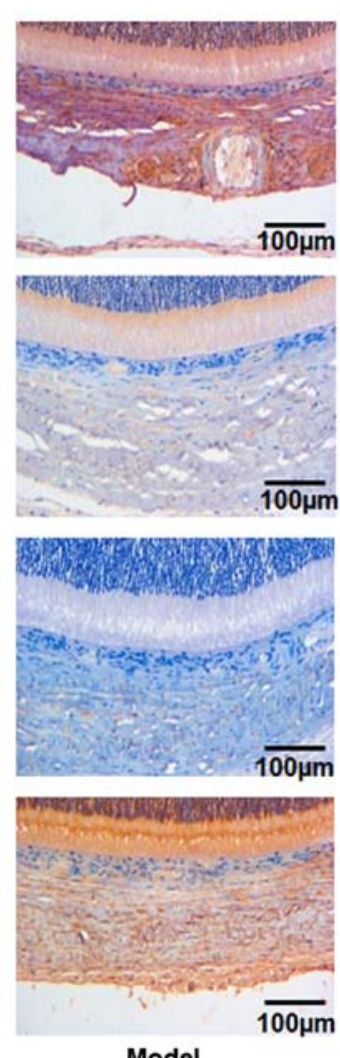

B
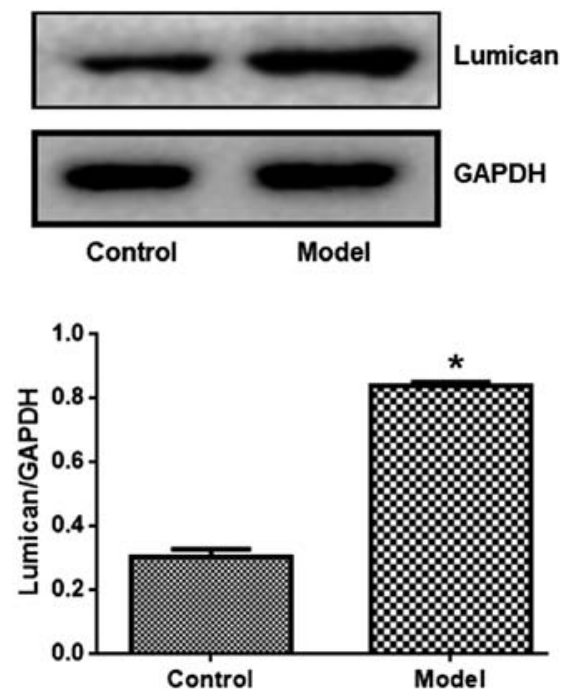

D

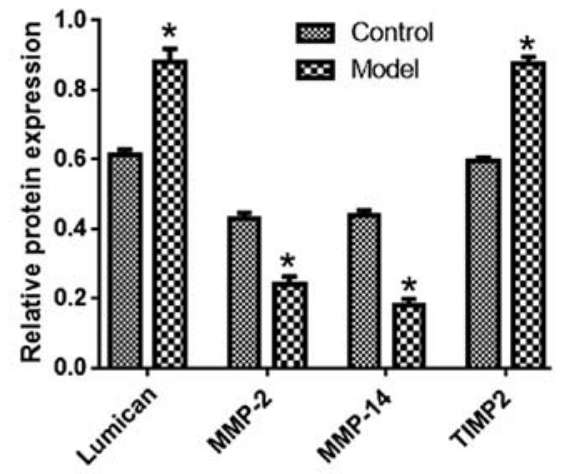

Figure 2. Expression of lumican, MMP-2, MMP-14 and TIMP-2 in scleral tissue. Expression levels of lumican, MMP-2, MMP-14 and TIMP-2 expression at the (A) mRNA and (B) protein levels, detected by reverse transcription-quantitative PCR and western blotting, respectively. (C) Immunohistochemical analysis of lumican, MMP-2, MMP-14 and TIMP-2 expression. (D) Quantification of results presented in panel C. * $\mathrm{P}<0.05$ vs. control eyes (paired t-tests). TIMP, tissue inhibitor of metalloproteinases.

\section{Discussion}

The results of the present study demonstrated that lumican and TIMP-2 were upregulated whereas MMP-2 and MMP-14 were downregulated in the scleral tissue of rats in a model of form-deprivation myopia. It was also revealed that overexpressing lumican in normal scleral fibroblasts induced apoptosis and reduced cell viability, which was associated with decreased MMP-2 and MMP-14 levels, as well as increased TIMP-2 expression. The results implied that elevated levels of lumican in scleral tissue contributed to the development of myopia.

The pathogenesis of myopia is mainly attributable to genetic factors (15) but is also related to the increase in diopter with age (16). Degeneration of the vitreous body may be caused by lengthening of the ocular axis and enlargement of the vitreous cavity (17). In the present study, a form-deprivation myopia model was successfully established, which was confirmed by an increased axial length and diopter, and histopathologic changes such as decreased scleral thickness. Ultrastructural analysis demonstrated that collagen fibers in the posterior sclera of model eyes were disorganized, with variable thickness and an enlarged space between fibers. These results indicated that myopia is associated with pathologic remodeling of collagen fibers in the sclera. Corneal thickness, anterior chamber depth or lens thickness was not examined in the present study due to technical limitations.

Lumican has been previously demonstrated to be overexpressed in a rat model of form-deprivation myopia (18). Lumican contributes to the formation of scleral collagen fibers; changes in fiber structure can lead to abnormal eye shape and size (19). Scleral remodeling involves extracellular matrix proteins and enzymes including collagen, proteoglycan and proteases (20). MMP-2 and TIMP-2 are typical proteases that serve an important role in scleral remodeling by regulating the balance between the synthesis and degradation of scleral fibroblast extracellular 
A

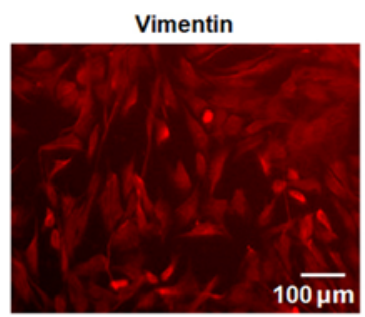

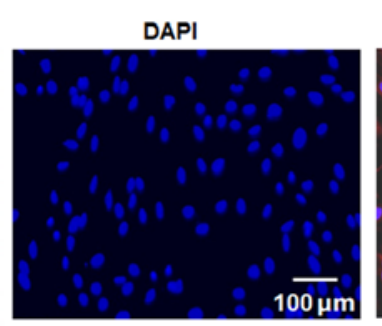

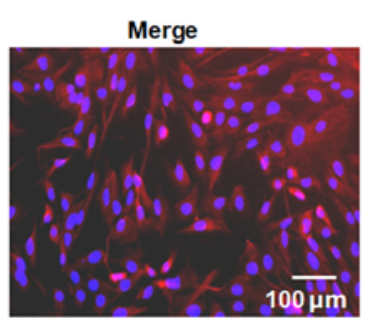

D
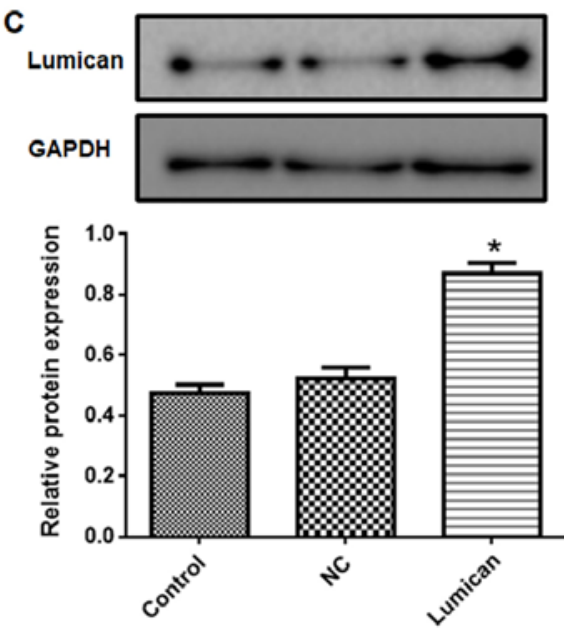

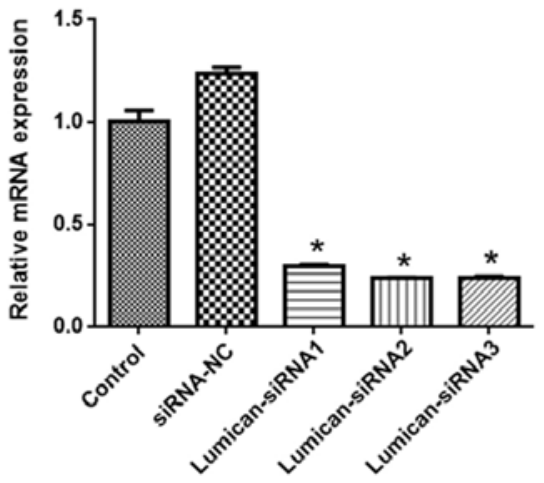

B

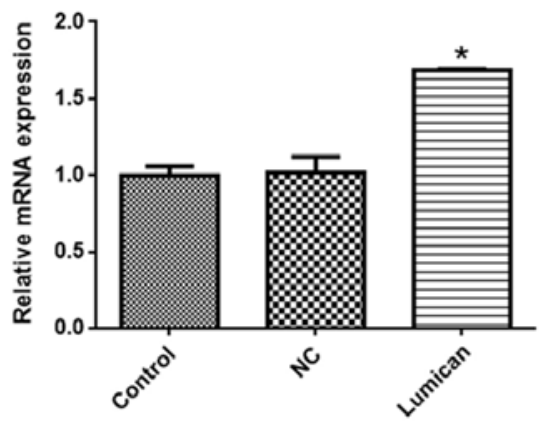

E
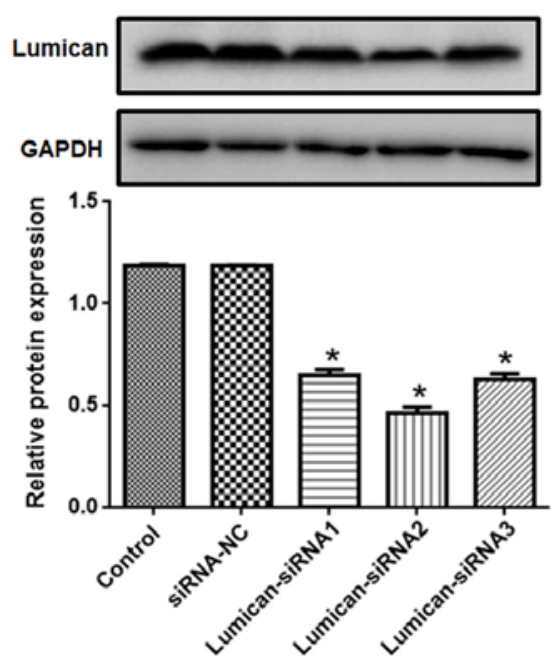

Figure 3. Effects of lumican overexpression and knockdown. (A) Vimentin expression were observed in cultured primary scleral fibroblasts. (B) mRNA and (C) protein expression levels of lumican in cells transfected with the lumican overexpression vector. (D) mRNA and (E) protein expression levels of lumican in cells transfected with lumican siRNA. "P<0.05 vs. the control group (one-way ANOVA and Tukey's post hoc tests). NC, negative control; si, small interfering.

matrix (21). MMP-2 was found to be upregulated in scleral tissue in a porcine model of form-deprivation myopia (22). Additionally, MMP-2 mRNA expression levels were increased, whereas TIMP-2 levels were decreased in the posterior scleral fiber layer in form-deprivation myopia (23). MMP-2 expression levels in sclera have also been demonstrated to be altered in a tree shrew myopia model (24). In chickens, an imbalance between MMP-2 and TIMP-2 levels is linked to abnormalities in the shape of the sclera (25). MMP-14 is expressed on the surface of scleral fibroblasts and directly degrades the extracellular matrix (26). MMP-2 is activated by MMP-14, which in turn activates MMP-2 by forming a complex with pro-MMP-2 and TIMP-2 (27).

Fibroblasts synthesize collagen fibers and other matrix components to form the outer wall of the eyeball. These cells maintain the integrity of eyeball tissue and determine the length of the ocular axis (28). The present study investigated the function of lumican in myopia using primary cultured scleral fibroblasts isolated from normal rat eyes. Most of the cells were vimentin ${ }^{+}$, indicating the successful isolation of scleral fibroblasts. Lumican expression has been demonstrated to alter collagen synthesis, leading to scleral remodeling, lengthening of the ocular axis and the development of myopia (29). Lumican binds to integrin on the cell surface to regulate cell proliferation and apoptosis (30). The present results revealed that proliferation was suppressed and apoptosis was enhanced in scleral fibroblasts by overexpressing lumican. Lumican induces apoptosis in endothelial cells through the activation of Fas/Fas ligand signaling, which suppresses integrin $\alpha 2 \beta 1$ activity and p38 MAPK signaling; reduces MMP-2 and MMP-14 expression, and increases that of TIMP-2, ultimately preventing the formation of the vascular lumen (22). Lumican is essential for promoting the growth and maintenance of corneal stromal transparency (31). Its overexpression has been demonstrated to reduce the expression of MMP-2 and MMP-14 in the corneal matrix and increase that of TIMP-2, thereby altering collagen fiber structure (32). Similarly, it was revealed that lumican overexpression decreased MMP-2 and MMP-14, and increased TIMP-2 expression in rat scleral fibroblasts, while the opposite effects were observed following lumican knockdown. These results are consistent with the role of TIMP-2 as a negative regulator of MMP-2 and indicated that this function may be perturbed in myopia, as suggested by previous studies $(27,32)$.

Inhibiting lumican expression may be an important mechanism for preventing apoptosis in scleral fibroblasts and stimulating MMP expression. In the present study, silencing lumican expression did not affect cell viability or apoptosis in scleral fibroblasts from normal rats although it altered TIMP-2 and MMP expression. These results suggested that the regulation of MMP expression is independent of fibroblast viability, which may be maintained by compensatory mechanisms in the absence of lumican.

In conclusion, the present results demonstrated that lumican overexpression promotes myopia by inducing apoptosis of 
A
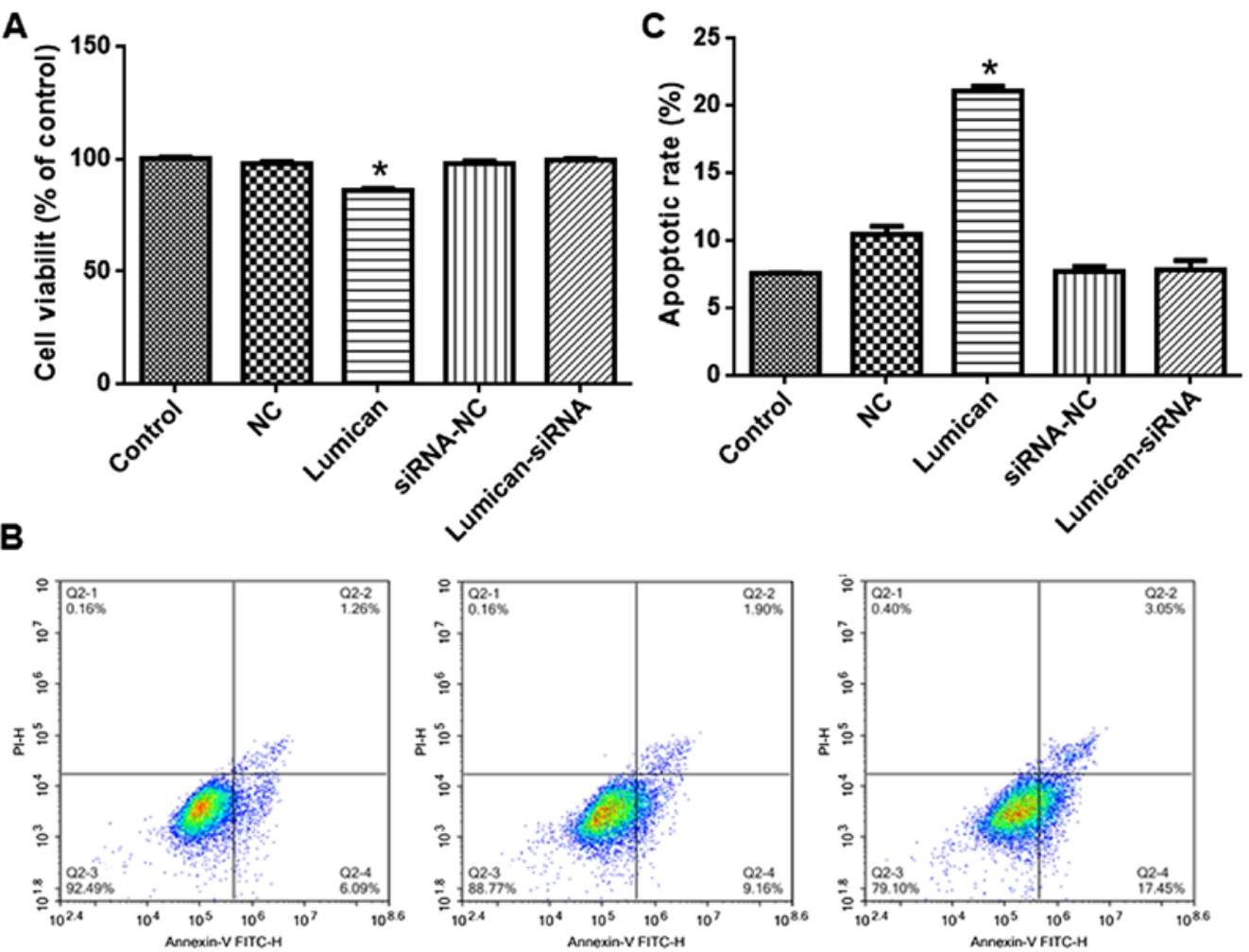

NC
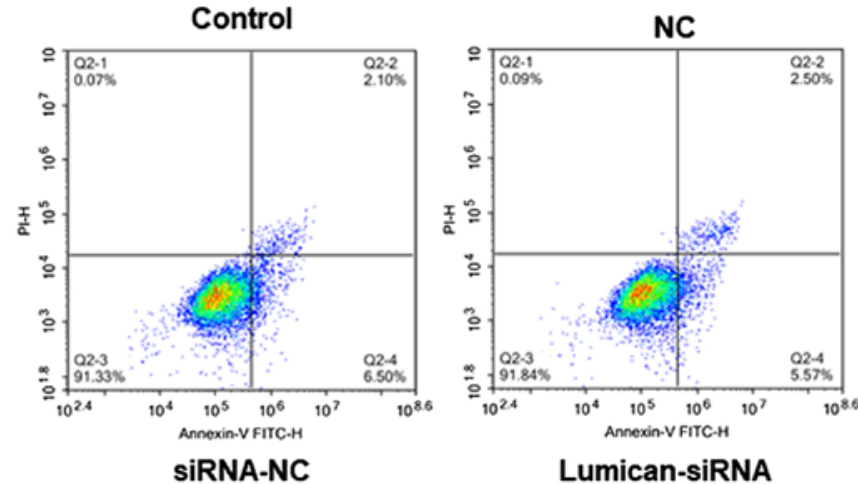

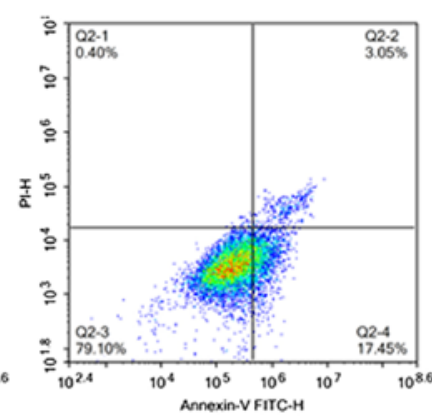

Lumican

Figure 4. Lumican overexpression reduces cell viability and promotes apoptosis in scleral fibroblasts. (A) Cell viability was evaluated using a Cell Counting Kit- 8 assay. (B) Apoptotic cells were detected using flow cytometry and (C) representative scatterplots were presented. ${ }^{*} \mathrm{P}<0.05$ vs. the control group (one-way ANOVA and Tukey's post hoc tests). NC, negative controls; si, small interfering.

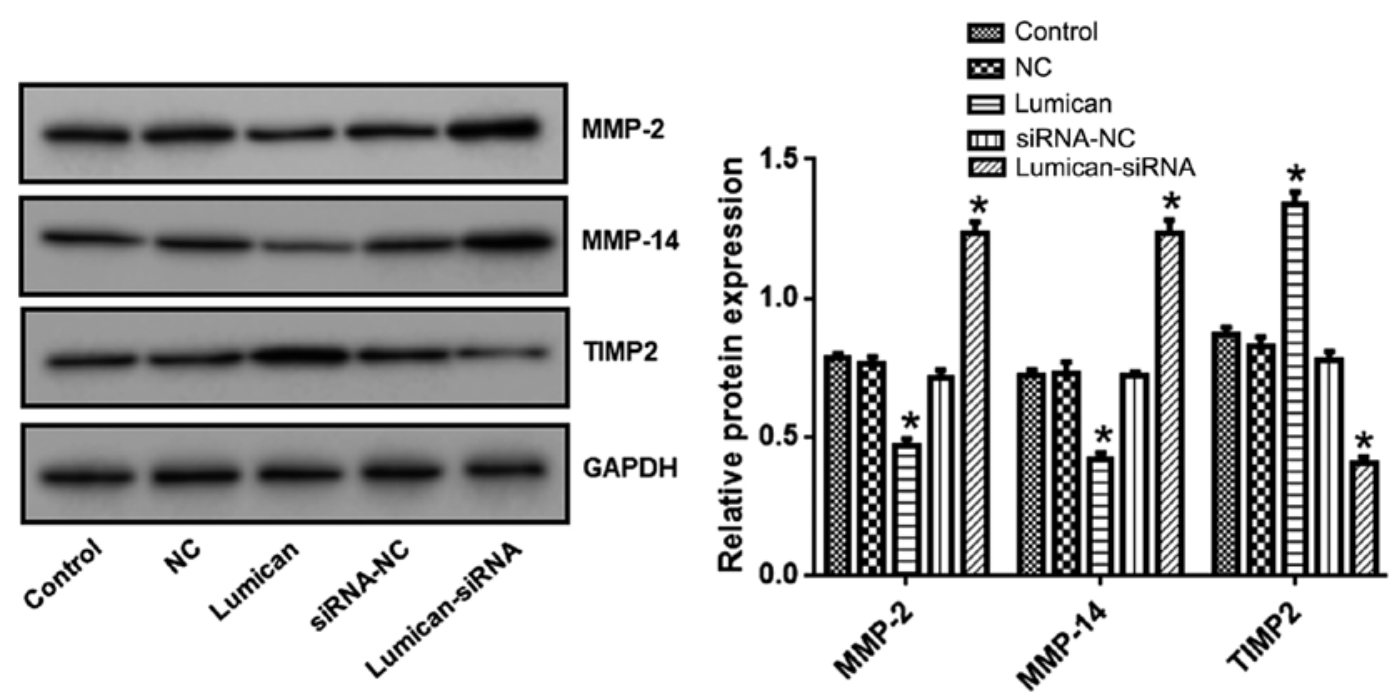

Figure 5. MMP-2, MMP-14 and TIMP-2 expression in rat scleral fibroblasts following lumican overexpression or knockdown. * $<0.05$ vs. the control group (one-way ANOVA and Tukey's post hoc tests). TIMP, tissue inhibitor of metalloproteinases. 
scleral fibroblasts and TIMP-2 expression and reducing MMP-2 and MMP-14 levels. As such, therapeutic strategies targeting lumican may be effective for the treatment of myopia.

\section{Acknowledgements}

Not applicable.

\section{Funding}

No funding was received.

\section{Availability of data and materials}

The datasets used and/or analyzed during the current study are available from the corresponding author on reasonable request.

\section{Authors' contributions}

JW, YZ, YF and SL performed the experiments and analyzed the data. JW and XZ designed the study and wrote the manuscript. All authors read and approved the final manuscript.

\section{Ethics approval and consent to participate}

All experimental protocol was approved by the Ethics Committee of the Second Affiliated Hospital of Nanchang University.

\section{Patient consent for publication}

Not applicable.

\section{Competing interests}

The authors declare that they have no competing interests.

\section{References}

1. Foster PJ and Jiang Y: Epidemiology of myopia. Eye (Lond) 28: 202-208, 2014

2. Hayashi M, Ito Y, Takahashi A, Kawano K and Terasaki H: Scleral thickness in highly myopic eyes measured by enhanced depth imaging optical coherence tomography. Eye (Lond) 27: 410-417, 2013.

3. Metlapally R and Wildsoet CF: Scleral mechanisms underlying ocular growth and myopia. Prog Mol Biol Transl Sci 134: 241-248, 2015.

4. Harper AR and Summers JA: The dynamic sclera: Extracellular matrix remodeling in normal ocular growth and myopia development. Exp Eye Res 133: 100-111, 2015.

5. Rada JA, Shelton S and Norton TT: The sclera and myopia. Exp Eye Res 82: 185-200, 2006.

6. Zhan X, Zhu ZC, Sun SQ and Wen YC: Dynamic changes of activator protein 1 and collagen I expression in the sclera of myopia guinea pigs. Int J Ophthalmol 12: 1272-1276, 2019.

7. Chen L, Zhang Y, Zuo Y, Ma F and Song H: Lumican expression in gastric cancer and its association with biological behavior and prognosis. Oncol Lett 14: 5235-5240, 2017.

8. Austin BA, Coulon C, Liu CY, Kao WW and Rada JA: Altered collagen fibril formation in the sclera of lumican-deficient mice. Invest Ophthalmol Vis Sci 43: 1695-1701, 2002.

9. Mouw JK, Ou G and Weaver VM: Extracellular matrix assembly: A multiscale deconstruction. Nat Rev Mol Cell Biol 15: 771-785, 2014.

10. Stuart K, Paderi J, Snyder PW, Freeman L and Panitch A: Collagen-binding peptidoglycans inhibit MMP mediated collagen degradation and reduce dermal scarring. PLoS One 6: e22139, 2011
11. Zhang P, Karani R, Turner RL, Dufresne C, Ferri S, Van Eyk JE and Semba RD: The proteome of normal human retrobulbar optic nerve and sclera. Proteomics 16: 2592-2596, 2016.

12. Xiao H, Fan ZY, Tian XD and Xu YC: Comparison of form-deprived myopia and lens-induced myopia in guinea pigs. Int J Ophthalmol 7: 245-250, 2014.

13. Livak KJ and Schmittgen TD: Analysis of relative gene expression data using real-time quantitative PCR and the 2(-Delta Delta C(T)) method. Methods 25: 402-408, 2001

14. Song ZJ, Yang SJ, Han L, Wang B and Zhu G: Postnatal calpeptin treatment causes hippocampal neurodevelopmental defects in neonatal rats. Neural Regen Res 14: 834-840, 2019.

15. Guggenheim JA, Ghorbani Mojarrad N, Williams C and Flitcroft DI: Genetic prediction of myopia: Prospects and challenges. Ophthalmic Physiol Opt 37: 549-556, 2017.

16. Steidl SM: How does visual acuity change over time in adults with high myopia? Br J Ophthalmol 90: 524, 2006.

17. Stone RA, Lin T, Laties AM and Iuvone PM: Retinal dopamine and form-deprivation myopia. Proc Natl Acad Sci USA 86: 704-706, 1989.

18. Sun MS, Song YZ, Zhang FJ, Tao J and Liu YB: Changes of ocular biological parameters and Lumican expression in the monocularly deprivation myopic model of mutant Lumican transgenic mice. Zhonghua Yan Ke Za Zhi 52: 850-855, 2016 (In Chinese).

19. Song Y, Zhang F, Zhao Y, Sun M, Tao J, Liang Y, Ma L, Yu Y, Wang $\mathrm{J}$ and Hao J: Enlargement of the axial length and altered ultrastructural features of the sclera in a mutant lumican transgenic mouse model. PLoS One 11: e0163165, 2016.

20. Lu P, Takai K, Weaver VM and Werb Z: Extracellular matrix degradation and remodeling in development and disease. Cold Spring Harb Perspect Biol 3: a005058, 2011.

21. Sternlicht MD and Werb Z: How matrix metalloproteinases regulate cell behavior. Annu Rev Cell Dev Biol 17: 463-516, 2001.

22. Vij N, Roberts L, Joyce S and Chakravarti S: Lumican suppresses cell proliferation and aids Fas-Fas ligand mediated apoptosis: Implications in the cornea. Exp Eye Res 78: 957-971, 2004.

23. Leung KH, Yiu WC, Yap MK, Ng PW, Fung WY, Sham PC and Yip SP: Systematic investigation of the relationship between high myopia and polymorphisms of the MMP2, TIMP2, and TIMP3 genes by a DNA pooling approach. Invest Ophthalmol Vis Sci 52: 3893-3900, 2011.

24. Siegwart JT Jr and Norton TT: Steady state mRNA levels in tree shrew sclera with form-deprivation myopia and during recovery. Invest Ophthalmol Vis Sci 42: 1153-1159, 2001.

25. Dai SZ, Zeng JW and Wang LY: Effect of pirenzepine on form deprivation myopia in chicks and its possible mechanism. Zhonghua Yan Ke Za Zhi 42: 42-47, 2006 (In Chinese).

26. Pietraszek K, Chatron-Colliet A, Brézillon S, Perreau C, Jakubiak-Augustyn A, Krotkiewski H, Maquart F and Wegrowski Y: Lumican: A new inhibitor of matrix metalloproteinase-14 activity. FEBS Lett 588: 4319-4324, 2014.

27. Lam C, Jamerson M, Cabral G, Carlesso AM and Marciano-Cabral F: Expression of matrix metalloproteinases in Naegleria fowleri and their role in invasion of the central nervous system. Microbiology (Reading) 163: 1436-1444, 2017.

28. Murata K, Hirata A, Ohta K, Enaida H and Nakamura KI: Morphometric analysis in mouse scleral fibroblasts using focused ion beam/scanning electron microscopy. Sci Rep 9: 6329, 2019.

29. Chen ZT, Wang IJ, Shih YF and Lin LL: The association of haplotype at the lumican gene with high myopia susceptibility in Taiwanese patients. Ophthalmology 116: 1920-1927, 2009.

30. Lu YP, Ishiwata $T$, Kawahara K, Watanabe M, Naito Z, Moriyama Y, Sugisaki Y and Asano G: Expression of lumican in human colorectal cancer cells. Pathol Int 52: 519-526, 2002.

31. Niewiarowska J, Brézillon S, Sacewicz-Hofman I, Bednarek R, Maquart F, Malinowski M, Wiktorska M, Wegrowski Y and Cierniewski CS: Lumican inhibits angiogenesis by interfering with alpha2beta1 receptor activity and downregulating MMP-14 expression. Thromb Res 128: 452-457, 2011.

32. Pietraszek K, Brézillon S, Perreau C, Malicka-Błaszkiewicz M, Maquart F and Wegrowski Y: Lumican-derived peptides inhibit melanoma cell growth and migration. PLoS One 8: e76232, 2013. This work is licensed under a Creative Commons
Attribution-NonCommercial-NoDerivatives 4.0
International (CC BY-NC-ND 4.0) License. 